\title{
Whole genome sequencing of Nontuberculous Mycobacterium (NTM) isolates from sputum specimens of co- habiting patients with NTM pulmonary disease and NTM isolates from their environment
}

\author{
Jung-Ki Yoon', Taek Soo Kim², Jong-II Kim ${ }^{3,4}$ and Jae-Joon Yim ${ }^{1 *}$
}

\begin{abstract}
Background: Nontuberculous mycobacterium (NTM) species are ubiquitous microorganisms. NTM pulmonary disease (NTM-PD) is thought to be caused not by human-to-human transmission but by independent environmental acquisition. However, recent studies using next-generation sequencing (NGS) have reported transcontinental spread of Mycobacterium abscessus among patients with cystic fibrosis.

Results: We investigated NTM genomes through NGS to examine transmission patterns in three pairs of cohabiting patients with NTM-PD who were suspected of patient-to-patient transmission. Three pairs of patients with NTM-PD co-habiting for at least 15 years were enrolled: a mother and a daughter with M. avium-PD, a couple with $M$. intracellulare-PD, and a second couple, one of whom was infected with $M$. intracellulare and the other of whom was infected with M. abscessus. Whole genome sequencing was performed using patients' NTM isolates as well as environmental specimens. Genetic distances were estimated based on single nucleotide polymorphisms (SNPs). By comparison with the genetic distances among 78 publicly available NTM genomes, NTM isolates derived from the two pairs of patients infected with the same NTM species were not closely related to each other. In phylogenetic analysis, the NTM isolates from patients with M. avium-PD clustered with isolates from different environmental sources.
\end{abstract}

Conclusions: In conclusion, considering the genetic distances between NTM strains, the likelihood of patient-topatient transmission in pairs of co-habiting NTM-PD patients without overt immune deficiency is minimal.

Keywords: Non-tuberculosis mycobacterium, Whole genome sequencing, Transmission, Non-tuberculous mycobacterial pulmonary disease, Phylogenomics

\footnotetext{
*Correspondence: yimjj@snu.ac.kr

'Division of Pulmonary and Critical Care Medicine, Department of Internal Medicine, Seoul National University College of Medicine, Seoul, Republic of Korea

Full list of author information is available at the end of the article
}

C C The Author(s). 2020 Open Access This article is licensed under a Creative Commons Attribution 4.0 International License, which permits use, sharing, adaptation, distribution and reproduction in any medium or format, as long as you give appropriate credit to the original author(s) and the source, provide a link to the Creative Commons licence, and indicate if changes were made. The images or other third party material in this article are included in the article's Creative Commons licence, unless indicated otherwise in a credit line to the material. If material is not included in the article's Creative Commons licence and your intended use is not permitted by statutory regulation or exceeds the permitted use, you will need to obtain permission directly from the copyright holder. To view a copy of this licence, visit http://creativecommons.org/licenses/by/4.0/. The Creative Commons Public Domain Dedication waiver (http://creativecommons.org/publicdomain/zero/1.0/) applies to the data made available in this article, unless otherwise stated in a credit line to the data. 


\section{Background}

The prevalence of nontuberculous mycobacterial pulmonary disease (NTM-PD) is increasing in developed countries [1-4]. Several explanations for this epidemiological change have been proposed, including awareness and improved detection of NTM-PD, increased populations with risk factors such as bronchiectasis or use of immunosuppressants, and disinfection of drinking water in urban areas resulting in selective advantages for NTM [1].

The development of next-generation sequencing (NGS) technology enables the identification of massive numbers of single nucleotide polymorphisms (SNPs) by whole genome sequencing (WGS). Using SNPs as genetic fingerprints and comparing them among multiple samples, phylogenetic analysis has been able to identify the source of infection for pathogens such as Vibrio cholera [5], Staphylococcus aureus [6], Pseudomonas aeruginosa [7], and NTM [8-11].

Since NTM are ubiquitous microorganism, it is generally assumed that patients with NTM-PD acquire NTM from their environment, not from other infected individuals. However, recent NGS studies showed that this might not be the case, at least for patients with cystic fibrosis [8, 9]. Bryant and colleagues collected Mycobacterium abscessus isolates from patients with cystic fibrosis, performed WGS, and analysed phylogenetic relationships among these isolates. Surprisingly, they found strong evidence supporting human-to-human transmission of $M$. abscessus among patients with cystic fibrosis, and identified some $M$. abscessus isolates that were widespread globally [9]. Although Harris and colleagues reported no evidence of patient-to-patient transmission in their cohort of pediatric cystic fibrosis patients [10], NGS studies of NTM transmission have raised concerns that NTM might be transmitted not only among immunosuppressed individuals but also among immunocompetent ones. This would be especially important for hospital infection control, since isolation practices for NTM-PD patients without cystic fibrosis are not as strict as those for pulmonary tuberculosis patients generally.

Recently, we diagnosed three pairs of NTM-PD patients with no immunodeficiency who had been cohabiting for at least 15 years. We investigated the genomes of NTM isolates derived from the patients and environmental samples in their houses to understand the source of infection using WGS.

\section{Results}

\section{Patient characteristics}

Three pairs of patients with NTM-PD who had been cohabiting for at least 15 years were enrolled (Table 1). A mother and a daughter (Patients $\mathrm{A}$ and $\mathrm{B}$ ) with $M$. avium $\mathrm{PD}$ had lived in an apartment in an urban area (HOME-1) for 15 years. A couple (Patients C and D) with $M$. intracellulare PD had lived in a house in a rural area (HOME-2) for 30 years. A second couple (Patients $\mathrm{E}$ and $\mathrm{F}$ ) with $M$. intracellulare $\mathrm{PD}$ and $M$. abscessus subsp. massiliense PD had lived in an apartment in an urban area (HOME-3) for 30 years. No patients were suspected of any immunodeficiency disorders. They were HIV-negative, were not taking any immunosuppressants and had no history of recurrent infection of any organs.

\section{NTM isolation and sequencing}

Among 12 environmental specimens from HOME-1, seven specimens from either the kitchen or the bathroom were culture-positive. Subsequently, 18 morphologically distinct isolates were purified (Supplementary

Table 1 Characteristics of the three patient pairs in this study

\begin{tabular}{|c|c|c|c|c|c|c|}
\hline & $\begin{array}{l}\text { Age } \\
\text { /Relationship }\end{array}$ & Sources & $\begin{array}{l}\text { NTM } \\
\text { Collection } \\
\text { Date }\end{array}$ & $\begin{array}{l}\text { NTM Species by } \\
\text { conventional } \\
\text { method }\end{array}$ & Habitat & Radiologic findings \\
\hline $\begin{array}{l}\text { Patient } \\
\text { A }\end{array}$ & $\begin{array}{l}81 \\
\text { /Mother }\end{array}$ & Sputum & $\begin{array}{l}5 \text { June } \\
2017\end{array}$ & M. avium & \multirow{2}{*}{$\begin{array}{l}\text { HOME-1 } \\
\text { Apartment (urban area) for } 15 \\
\text { years. }\end{array}$} & $\begin{array}{l}\text { Bronchiectasis and centrilobular nodules in } \\
\text { RML/LUL lingular segments }\end{array}$ \\
\hline $\begin{array}{l}\text { Patient } \\
\text { B }\end{array}$ & $\begin{array}{l}51 \\
\text { /Daughter }\end{array}$ & Sputum & $\begin{array}{l}19 \text { July } \\
2017\end{array}$ & M. avium & & $\begin{array}{l}\text { Centrilobular nodules with branching } \\
\text { opacity in RUL/RML/LUL lingula segments }\end{array}$ \\
\hline $\begin{array}{l}\text { Patient } \\
\text { C }\end{array}$ & $\begin{array}{l}77 \\
\text { /Husband }\end{array}$ & Sputum & $\begin{array}{l}13 \text { April } \\
2017\end{array}$ & M. intracellulare & \multirow{2}{*}{$\begin{array}{l}\text { HOME-2 } \\
\text { House (rural area) for } 30 \text { years } \\
\text { with high soil environment }\end{array}$} & Lung nodule in RUL \\
\hline $\begin{array}{l}\text { Patient } \\
\text { D }\end{array}$ & $\begin{array}{l}71 \\
\text { Mife }\end{array}$ & Sputum & 3 May 2017 & M. intracellulare & & $\begin{array}{l}\text { Multiple branching opacity and } \\
\text { centrilobular nodules in both lung }\end{array}$ \\
\hline $\begin{array}{l}\text { Patient } \\
\text { E }\end{array}$ & $\begin{array}{l}62 \\
\text { /Husband }\end{array}$ & $\begin{array}{l}\text { Bronchial } \\
\text { washing }\end{array}$ & $\begin{array}{l}29 \\
\text { September } \\
2017\end{array}$ & M. intracellulare & \multirow[t]{2}{*}{$\begin{array}{l}\text { HOME-3 } \\
\text { Apartment (urban area) for } 30 \\
\text { years. }\end{array}$} & $\begin{array}{l}\text { Bronchiectasis with peribonchial infiltration } \\
\text { in LLL }\end{array}$ \\
\hline $\begin{array}{l}\text { Patient } \\
\text { F }\end{array}$ & $\begin{array}{l}61 \\
\text { Mife }\end{array}$ & $\begin{array}{l}\text { Bronchial } \\
\text { washing }\end{array}$ & $\begin{array}{l}29 \\
\text { September } \\
2017\end{array}$ & $\begin{array}{l}\text { M. abscessus subsp. } \\
\text { massiliense }\end{array}$ & & Patchy opacity and nodules in RML \\
\hline
\end{tabular}


Table 1, Additional File 1). However, none of the 15 environmental specimens from HOME-2 yielded any NTM isolates and only one of 15 specimens from HOME-3 yielded NTM isolates.

On average, 19.8 million sequencing reads were obtained for each isolate (See Supplementary Table 2, Additional File 1). According to $k$-mer based taxonomic classification of NGS reads, 12 isolates from environmental specimens in HOME-1, isolates from Patient A and Patient B were identified as $M$. avium subsp. hominissuis. Isolates from Patients C, D, and E were identified as $M$. intracellulare, and one isolate from Patient $F$ was identified as $M$. abscessus. The identifications of patientderived isolates using NGS produced the same results as conventional PCR and direct sequencing. Two isolates from environmental specimens in HOME-1 were identified as $M$. fortuitum, while the remaining four isolates from HOME-1 and three isolates from HOME-3 were classified as non-NTM and were excluded.

The mean sequencing depth of identified isolates was $313 \times(210-487 \times)$. To reduce the potential impact of recombination and mobile genetic elements on our results, we defined core regions for each species as common sequences observed across the $17 \mathrm{M}$. avium subsp. hominissuis and $3 \mathrm{M}$. intracellulare genomes analyzed in this study (as well as 64 and 14 publicly available genomes, respectively). The core region of $M$. avium subsp. homonissuis consisted of $4.30 \mathrm{Mbp}$ of the $5.15 \mathrm{Mbp}$ genome (83\%), and that of $M$. intracellulare consisted of 4.23 Mbp of the 5.40 Mbp genome (78\%). On average, 38,377 $(27,469-43,720)$ high confidence SNPs were detected in M. avium subsp. hominissuis and 16,464 (15-26,740) high confidence SNPs were detected in M. intracellulare within these regions (Supplementary Table 2, Additional File 1). Based on the distributions of SNP allele fraction (Supplementary Table 2, Additional File 1), all isolates were monoclonal except for the isolate from $\mathrm{Pa}$ tient $\mathrm{C}$, which consisted of two clones at an 8:2 ratio (Supplementary Figure 1). The 25,441 and 2485 high confidence SNPs from Patient $\mathrm{C}$ were classified as belonging to the Cmajor and Cminor clones based on SNP allele fractions.

\section{Pairwise SNP distances and phylogenetic analysis}

A total of 104,531 and 102,281 genomic positions with high-confidence SNPs were identified in M. avium subsp. hominissuis and $M$ intracellulare genome, respectively, and used for further analysis. Pairwise SNP distances between every pair of isolates at those positions were calculated and clusters on the histogram of SNP distances were observed (Fig. 1). The SNP distance between the isolates from Patient A and B was 14,768. By contrast, the SNP distances among three replicates (the isolates from Patient A, Kitchen Sink Faucet 1, and Kitchen Sink Cold Water 3) were less than 100. (Fig. 1a) Using high-confidence SNPs from 81M. avium subsp. hominissuis genomes, phylogenetic analysis was performed (Fig. 2a). The isolate from Patient A and its replicates clustered with the specimens from the kitchen (scale on surface of kitchen faucet), while the isolate from Patient B clustered with the isolates from the bathroom (hot water from bathroom faucet, hot water from showerhead) and the kitchen (cold water from kitchen faucet). (a) M. avium supsp. homonissuis

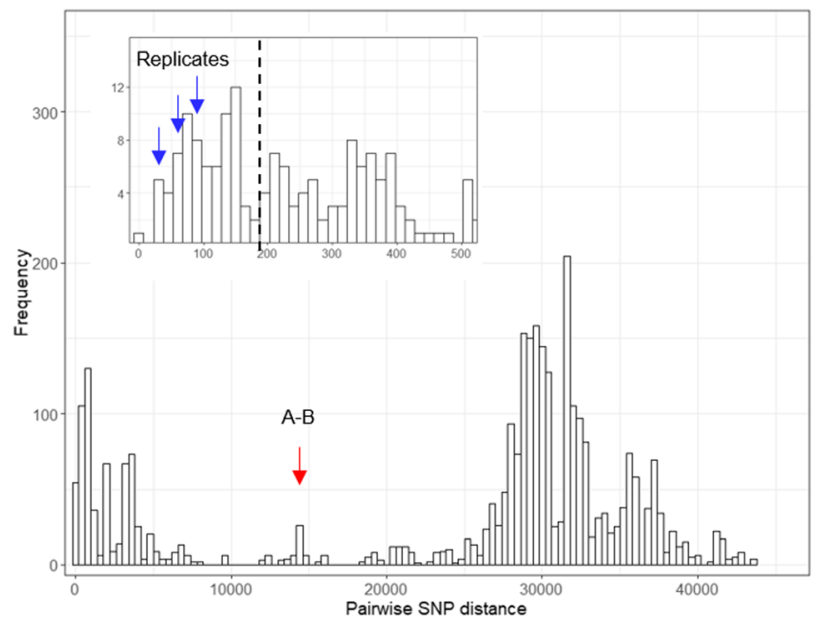

(b) $M$. intracellulare

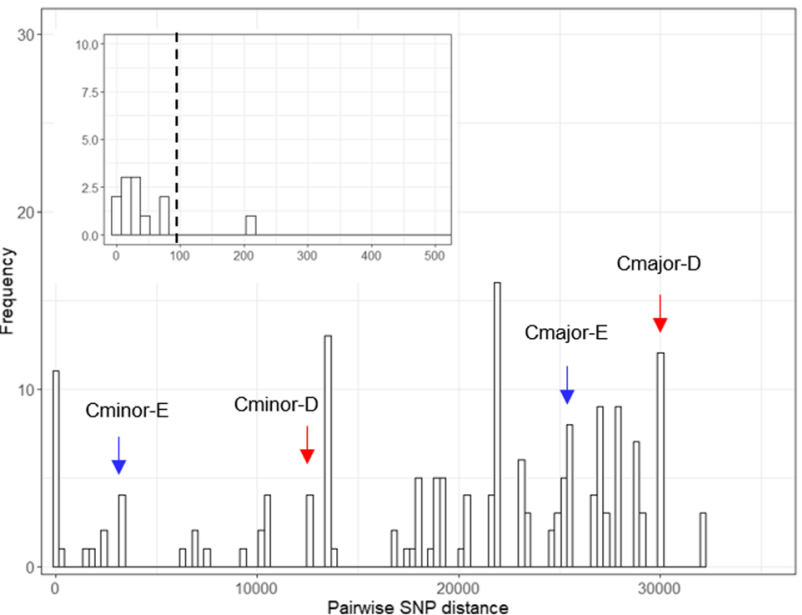

Fig. 1 Histograms of pairwise SNP distances. The $x$-axis shows pairwise SNP distance and the $y$-axis shows the frequencies of distances between isolates. (a) Distances between replicates of M. avium subsp. hominissuis (blue arrow) were less than 200 (black dashed line), whereas the distance between isolates from Patient A and B (red arrow) was 14,768. (b) The distance between $M$. intracellulare isolates from Patients C and D living together in HOME-2 (red arrow) was higher than the distance between isolates from Patients $\mathrm{C}$ and $\mathrm{E}$ living in other houses (blue arrow) 


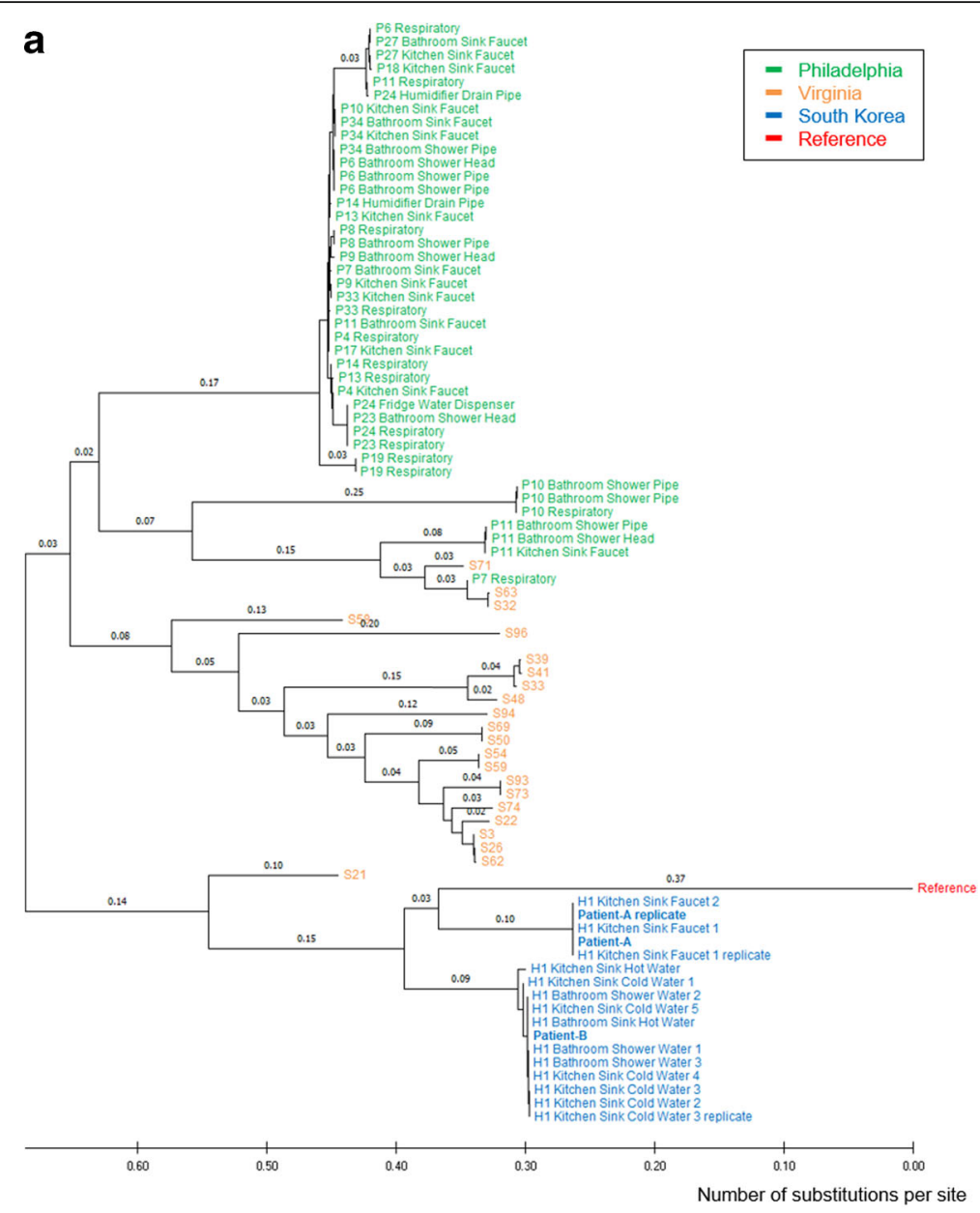

b

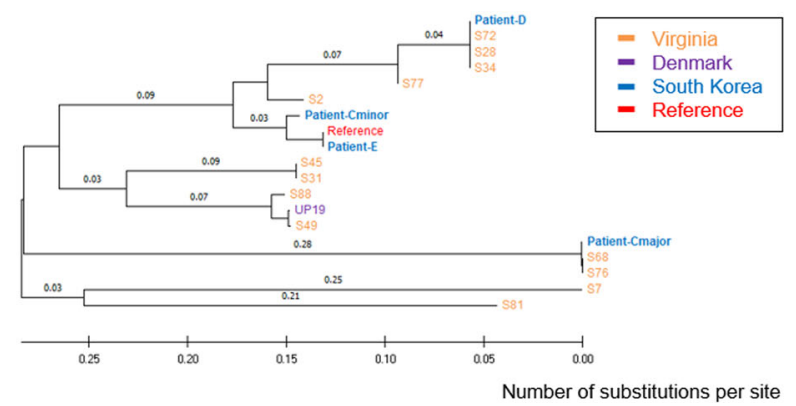

Fig. 2 Phylogenetic analysis of M. avium subsp. homonissuis and M. intracellulare isolates. A neighbor-joining phylogenetic tree was drawn to scale with branch lengths in units of number of base substitutions per site. (a) Isolates from Patients A and B (blue) clustered apart from environmental isolates from HOME-1, and 64 publicly available M. avium subsp. hominissuis genome sequences clustered as expected based on previously published work. (b) Isolates from Patients C and D (blue) clustered separately from other isolates

In HOME-2, the SNP distance between the M. intracellulare isolates from Patients Cmajor and D was 29, 873, and the distance between Patient Cminor and D was 12,670. Those distances were even higher than the SNP distances from Patient E in HOME-3. (Fig. 1b) Phylogenetic analysis with $17 \mathrm{M}$. intracellulare genomes confirmed that all three isolates from Patient C, D, and $\mathrm{E}$ were not closely related each other. (Fig. 2b) In HOME-3, different species of NTM were isolated ( $M$. intracellulare from Patient E and M. abscessus subsp. massiliense from patient F), and the SNP distance was not calculated. 


\section{Discussion}

Recent NGS studies suggest the patient-to-patient transmission of $M$. abscessus among cystic fibrosis patients $[8,9]$. In response to these findings, the NTM guidelines have been updated. The British Thoracic Society [12], US Cystic Fibrosis Foundation and European Cystic Fibrosis Society [13] recommended that infection control policies minimize risks of patient-to-patient transmission for patients with both cystic fibrosis and $M$. abscessusPD. However, these guidelines were only designed for patients with underlying risk factors and contain no statements for patients without immunodeficiencies. In this context, it is clinically important to understand if there is any evidence of patient-to-patient transmission among patients without predisposing factors.

Among the three pairs of co-habiting NTM-PD patients without overt immunodeficiency examined here, no patient pairs had near-identical NTM isolates (Fig. 2). A pair of patients were infected with M. avium-PD isolates that were genetically distinct but similar to separate isolates from environmental specimens in their homes. Although we could not identify environmental sources for the remaining two pairs of patients, one patient pair was also infected with genetically distinct $M$. intracellulare isolates (SNP distance $>10^{4}$ ), and the last pair of patients was infected by different species of NTM (M. intracellulare and M. abscessus subsp. massiliense). Therefore, patient-to-patient transmission is less likely in our study.

Household water sources are considered a major reservoir for NTM, especially for $M$. avium. One report showed that samples from 17 (46\%) of 37 households yielded the same species of NTM found in the patient. In seven of those households, the patient isolate and a plumbing isolate exhibited the same repetitive sequencebased PCR DNA fingerprint. Another study reported that seven of 20 (35\%) patients with NTM-PD had NTM isolates with identical genotypes to isolates from household water or shower aerosols [14]. Recently, variable number tandem repeat genotyping and WGS revealed that 11 of 21 (52\%) patients with M. avium-PD had genetically matched isolates to household isolates [11]. In our study, a phylogenetic tree based on WGS was consistent with these previous reports and demonstrated the environmental sources of two M. avium-PD patients living together (HOME-1). Patient A seemed to have acquired NTM from the kitchen, while Patient B acquired NTM from the bathroom or kitchen.

Although we thoroughly collected environmental specimens from water and biofilms on the faucets and showerhead, we could not culture any NTMs from HOME-2, and identified only one NTM from HOME-3. A previous study reported that NTM species could be isolated from 22 of 37 (59\%) households of NTM-PD patients. In addition, a positive correlation was observed between the number of samples collected per house and the number of NTM-positive samples [15]. We collected 15 water samples from each of the two houses; this number is equal to or larger than that of most studies, suggesting that there may be sources of NTM-PD other than water. The patients living in HOME-2 (Patient $\mathrm{C}$ and $\mathrm{D}$ ) were farmers and their home was a high soil environment that included a yard and hay (Table 1). Given that previous studies reported that a majority of $M$. intracellulare was isolated from soil samples, especially in high soil environments $[14,16,17]$, the source of $M$. intracellulare for these patients could be soil instead of water.

In HOME-3, three morphologically distinct colonies were cultured from one environmental specimen and sequenced separately. However, using $k$-mer based taxonomic classification, these isolates were not NTM. The sequencing reads of NTM isolates from Patients E and F aligned to NTM reference genomes, but those from environmental specimen isolates did not. Thus, these environmental isolates were not closely related to NTM in HOME-3. A previous study showed that, among 19 patients with $M$. avium-PD or $M$. intracellulare-PD living in low soil environments, no patients had genetically identical isolates compared with soil sample isolates from their houses [17]. Our results suggested that a patient with $M$. intracellulare-PD (Patient E) in HOME-3 (low soil) may have been exposed to the NTM outside the house. The lag time between the acquisition of NTM from the environment and diagnosis of NTM-PD also makes it more difficult to identify the source of NTM [14].

\section{Conclusions}

NTM isolates derived from three pairs of co-habiting NTM-PD patients were determined to be different strains based on WGS data. The sources of M. avium in patients in one pair was identified: one acquired infection from the kitchen and the other from the bathroom or kitchen. The likelihood of patient-to-patient transmission appeared minimal in these three pairs of NTM-PD patients.

\section{Methods}

\section{Patient enrolment}

Pairs of adult patients living together and diagnosed with NTM-PD at Seoul National University Hospital satisfying the following inclusion criteria were enrolled: age > 18 years; typical respiratory symptoms such as chronic cough, sputum, or haemoptysis; findings suggestive of NTM-PD on computed tomography; identification of NTM in $\geq 2$ sputum cultures or in $\geq 1$ bronchoscopic specimen; living in the same household prior to diagnosis with NTM-PD; and consented to collection of 
environmental samples in their home. This study was approved by an institutional review board (IRB Number: 1804-064-936) and registered at ClinicalTrial.gov (NCT03532438).

\section{Sample collection and NTM isolation}

The most recently-isolated NTM from participant sputum or bronchial wash specimens from participants was collected. In addition, we visited patients' homes to collect environmental specimens. One litre of water was aseptically collected into sterile containers directly from all faucets and showers in bathrooms and kitchens. Swabs were taken from the inside of faucets and showerheads in bathrooms and kitchens. As previously described [14, 18], each water specimen was passed through a $0.45 \mu \mathrm{m}$ filter. Filters were rinsed with $10 \mathrm{~mL}$ of sterile distilled water and transferred to Middlebrook 7H11 plates and Mycobacterial Growth Indicator Tubes (MGITs). Swabs were washed in sterile distilled water and then processed in the same manner as the water samples. All culture-positive MGITs were transferred to new Middlebrook $7 \mathrm{H} 11$ plates. If culture-positive Middlebrook $7 \mathrm{H} 11$ plates had two or more morphologically distinct colonies in terms of size and color, each distinct colony was separately transferred to a $3 \%$ Ogawa media plate, incubated, and purified as a single colony. If the colonies were homogenous and discrete on Middlebrook $7 \mathrm{H} 11$ plates, we collected the isolates without a further purification step.

\section{DNA preparation and sequencing}

All biomass from Middlebrook 7H11 plates or Ogawa media plates taken from sweeps of colonies was mixed with $425-600 \mu \mathrm{m}$ glass beads, vortexed for $5 \mathrm{~min}$, incubated at $80^{\circ} \mathrm{C}$ for $10 \mathrm{~min}$ and then centrifuged. DNA was extracted from the supernatant using a QIAamp DNA mini kit (Qiagen inc, Hilden, Germany) as previously described [8]. Subsequently, DNA was sequenced using an Illumina HiSeq or NovaSeq instrument (Illumina, San Diego, USA). To validate the method, two patient isolates and an environmental specimen isolate were sequenced twice as replicates. Identification of each isolate from the patients was performed by $16 \mathrm{~S}$ rRNA [19] and rpoB gene [20,21] sequencing analysis.

\section{Variant calling and phylogenetic analysis}

Kraken2, a taxonomic classification system using $k$-mer matches [22], was used to identify reads from each isolate mapping to reference genomes. For isolates assigned as $M$. avium subsp. homonissius, reads were mapped to M. avium subsp. homonissius TH15 using BWA [23]. For isolates assigned as $M$. intracellulare, reads were mapped to $M$. intracellulare ATCC 13950. Isolates assigned by Kraken2 as non-NTM species were excluded from further analyses. Variants were called using SAMtools and bcftools [24] with the following filters: minimum base quality of 50, minimum mapping quality of 30 , support from at least four reads (two forward reads, two reverse reads), and absence of heterozygosity. For comparison, $64 \mathrm{M}$. avium subsp. homonissius and $14 \mathrm{M}$. intracellulare genomes available in three publicly available datasets deposited in the short read archive (PRJNA339271, PRJNA506132, PRJEB13214) were downloaded and processed using the same procedures $[11,25,26]$. Core regions were defined for each NTM species as all genomic positions with depths from all isolates of each species of mean depth \pm two standard deviations. High-confidence SNPs were defined as SNPs in the core regions of each NTM species. SNP distance and the number of different high-confidence SNPs were calculated for each pair of isolates. The phylogenetic analysis was conducted with MEGA-X (version 10.0.5) [27] using only high confidence SNPs. The genetic distances between isolates were computed using the maximum composite likelihood method and a phylogenetic tree was constructed using neighbor-joining methods. The rate variation among sites was modeled with a gamma distribution (shape parameter $=1$ ). Reliability of the phylogeny was assessed using the bootstrap test (number of bootstrap replicates $=100$ ). All sequence data was deposited in the National Center for Biotechnology Information database as BioProject ID PRJNA577108.

\section{Supplementary information}

Supplementary information accompanies this paper at https://doi.org/10. 1186/s12864-020-6738-2

Additional file 1: Table S1. Characteristics on environmental specimens. The numbers of collected specimens, culture-positive, morphologically distinct isolates from each habitat. Table S2. NTM species Identified and Sequencing Results. Description on the source of each sample and its sequencing quality (total read counts, mean depth, 20x coverage, number of SNPS), species, clonality, and morphologic feature.

Additional file 2

\section{Abbreviations}

MGIT: Mycobacterial growth indicator tubes; NGS: Next-generation sequencing; NTM: Non-tuberculous mycobacterium; NTM-PD: Nontuberculous mycobacterial pulmonary disease; SNP: Single nucleotide polymorphism; WGS: Whole genome sequencing

\section{Acknowledgements}

Not applicable.

\section{Authors' contributions}

J-JY designed the experiments. J-KY and J-JY participated in data collection. TSK isolated the bacteria. J-KY, TSK, J-IK and J-JY analyzed the data and wrote the paper. J-IK and J-JY supervised the study. All the authors read and approved the final manuscript.

Funding

Not applicable. 


\section{Availability of data and materials}

All sequence data supporting the conclusions of this article is available in the National Center for Biotechnology Information database as BioProject ID PRJNA577108.

\section{Ethics approval and consent to participate}

All participants were informed about the study and written consent was obtained prior to the enrollment. Ethical approval of this study was approved by the institutional review board of Seoul National University Hospital (IRB Number: 1804-064-936).

\section{Consent for publication}

Not Applicable.

\section{Competing interests}

The authors declare that they have no competing interests.

\section{Author details}

'Division of Pulmonary and Critical Care Medicine, Department of Internal Medicine, Seoul National University College of Medicine, Seoul, Republic of Korea. ${ }^{2}$ Department of Laboratory Medicine, Seoul National University Hospital, Seoul, Republic of Korea. ${ }^{3}$ Department of Biomedical Sciences, Seoul National University Graduate School, Seoul, Republic of Korea. ${ }^{4}$ Genomic Medicine Institute, Medical Research Center, Seoul National University School, Seoul, Republic of Korea.

\section{Received: 19 November 2019 Accepted: 15 April 2020}

\section{Published online: 23 April 2020}

\section{References}

1. Adjemian J, Olivier KN, Seitz AE, Holland SM, Prevots DR. Prevalence of nontuberculous mycobacterial lung disease in U.S. Medicare beneficiaries. Am J Respir Crit Care Med. 2012;185(8):881-6.

2. Park YS, Lee CH, Lee SM, Yang SC, Yoo CG, Kim YW, et al. Rapid increase of non-tuberculous mycobacterial lung diseases at a tertiary referral hospital in South Korea. Int J Tuberc Lung Dis. 2010:14(8):1069-71.

3. Izumi K, Morimoto K, Hasegawa N, Uchimura K, Kawatsu L, Ato M, et al. Epidemiology of adults and children treated for Nontuberculous mycobacterial pulmonary disease in Japan. Ann Am Thorac Soc. 2019; 16(3):341-7.

4. Lee H, Myung W, Koh WJ, Moon SM, Jhun BW. Epidemiology of Nontuberculous mycobacterial infection, South Korea, 2007-2016. Emerg Infect Dis. 2019:25(3):569-72.

5. Chin CS, Sorenson J, Harris JB, Robins WP, Charles RC, Jean-Charles RR et al. The origin of the Haitian cholera outbreak strain. $\mathrm{N}$ Engl J Med. 2011;364(1):33-42

6. Harris SR, Feil EJ, Holden MT, Quail MA, Nickerson EK, Chantratita N, et al. Evolution of MRSA during hospital transmission and intercontinental spread. Science. 2010;327(5964):469-74.

7. Quick J, Cumley N, Wearn CM, Niebel M, Constantinidou C, Thomas CM, et al. Seeking the source of Pseudomonas aeruginosa infections in a recently opened hospital: an observational study using whole-genome sequencing. BMJ Open. 2014;4(11):e006278.

8. Bryant JM, Grogono DM, Greaves D, Foweraker J, Roddick I, Inns T, et al. Whole-genome sequencing to identify transmission of Mycobacterium abscessus between patients with cystic fibrosis: a retrospective cohort study. Lancet. 2013;381(9877):1551-60.

9. Bryant JM, Grogono DM, Rodriguez-Rincon D, Everall I, Brown KP, Moreno P, et al. Emergence and spread of a human-transmissible multidrug-resistant nontuberculous mycobacterium. Science. 2016:354(6313):751-7.

10. Harris KA, Underwood A, Kenna DT, Brooks A, Kavaliunaite E, Kapatai G, et al. Whole-genome sequencing and epidemiological analysis do not provide evidence for cross-transmission of mycobacterium abscessus in a cohort of pediatric cystic fibrosis patients. Clin Infect Dis. 2015;60(7):1007-16.

11. Lande L, Alexander DC, Wallace RJ Jr, Kwait R, lakhiaeva E, Williams M, et al. Mycobacterium avium in community and household water, suburban Philadelphia, Pennsylvania, USA, 2010-2012. Emerg Infect Dis. 2019;25(3): 473-81

12. Haworth CS, Banks J, Capstick T, Fisher AJ, Gorsuch T, Laurenson IF et al. British Thoracic Society guidelines for the management of non- tuberculous mycobacterial pulmonary disease (NTM-PD). Thorax. 2017; 72(Suppl 2):ii 1-ii64.

13. Floto RA, Olivier KN, Saiman L, Daley CL, Herrmann JL, Nick JA, et al. US Cystic Fibrosis Foundation and European cystic fibrosis society consensus recommendations for the management of non-tuberculous mycobacteria in individuals with cystic fibrosis. Thorax. 2016;71(Suppl 1):i1-22.

14. Thomson R, Tolson C, Carter R, Coulter C, Huygens F, Hargreaves M. Isolation of nontuberculous mycobacteria (NTM) from household water and shower aerosols in patients with pulmonary disease caused by NTM. J Clin Microbiol. 2013;51(9):3006-11.

15. Falkinham JO 3rd. Nontuberculous mycobacteria from household plumbing of patients with nontuberculous mycobacteria disease. Emerg Infect Dis. 2011;17(3):419-24

16. Feazel LM, Baumgartner LK, Peterson KL, Frank DN, Harris JK, Pace NR. Opportunistic pathogens enriched in showerhead biofilms. Proc Natl Acad Sci U S A. 2009;106(38):16393-9.

17. Fujita K, Ito Y, Hirai T, Maekawa K, Imai S, Tatsumi S, et al. Genetic relatedness of Mycobacterium avium-intracellulare complex isolates from patients with pulmonary MAC disease and their residential soils. Clin Microbiol Infect. 2013;19(6):537-41.

18. Thomson RM, Carter R, Tolson C, Coulter C, Huygens F, Hargreaves M. Factors associated with the isolation of Nontuberculous mycobacteria (NTM) from a large municipal water system in Brisbane. Austr BMC Microbiol. 2013;13:89.

19. Petti CA, Clinical and Laboratory Standards Institute. Interpretive criteria for identification of bacteria and fungi by DNA target sequencing : approved guideline, vol. viii. Wayne: Clinical and Laboratory Standards Institute; 2008. p. 73

20. Kim BJ, Lee SH, Lyu MA, Kim SJ, Bai GH, Chae GT, et al. Identification of mycobacterial species by comparative sequence analysis of the RNA polymerase gene (rpoB). J Clin Microbiol. 1999;37(6):1714-20.

21. Ben Salah I, Adekambi T, Raoult D, Drancourt M. rpoB sequence-based identification of Mycobacterium avium complex species. Microbiology. 2008; 154(Pt 12):3715-23.

22. Wood DE, Lu J, Langmead B. Improved metagenomic analysis with kraken 2. Genome Biol. 2019;20(1):257

23. Li H, Durbin R. Fast and accurate short read alignment with burrowswheeler transform. Bioinformatics. 2009;25(14):1754-60.

24. Li H. A statistical framework for SNP calling, mutation discovery, association mapping and population genetical parameter estimation from sequencing data. Bioinformatics. 2011;27(21):2987-93.

25. Operario DJ, Pholwat S, Koeppel AF, Prorock A, Bao Y, Sol-Church K, et al. Mycobacterium avium complex diversity within lung disease, as revealed by whole-genome sequencing. Am J Respir Crit Care Med. 2019:200(3):393-6.

26. van Ingen J, Kohl TA, Kranzer K, Hasse B, Keller PM, Katarzyna Szafranska A et al. Global outbreak of severe Mycobacterium chimaera disease after cardiac surgery: a molecular epidemiological study. Lancet Infect Dis. 2017; 17(10):1033-41.

27. Kumar S, Stecher G, Li M, Knyaz C, Tamura K, MEGA X. Molecular evolutionary genetics analysis across computing platforms. Mol Biol Evol. 2018:35(6):1547-9.

\section{Publisher's Note}

Springer Nature remains neutral with regard to jurisdictional claims in published maps and institutional affiliations.

Ready to submit your research? Choose BMC and benefit from:

- fast, convenient online submission

- thorough peer review by experienced researchers in your field

- rapid publication on acceptance

- support for research data, including large and complex data types

- gold Open Access which fosters wider collaboration and increased citations

- maximum visibility for your research: over $100 \mathrm{M}$ website views per year

At BMC, research is always in progress.

Learn more biomedcentral.com/submission 